\title{
ON ASYMPTOTIC CRITICAL VALUES AND THE RABIER THEOREM
}

\author{
ZBIGNIEW JELONEK \\ Institute of Mathematics, Polish Academy of Sciences \\ Św. Tomasza 30, 31-027 Kraków, Poland \\ E-mail: najelone@cyf-kr.edu.pl
}

\begin{abstract}
Let $X \subset k^{n}$ be a smooth affine variety of dimension $n-r$ and let $f=\left(f_{1}, \ldots, f_{m}\right)$ : $X \rightarrow k^{m}$ be a polynomial dominant mapping. It is well-known that the mapping $f$ is a locally trivial fibration outside a small closed set $B(f)$. It can be proved (using a general Fibration Theorem of Rabier) that the set $B(f)$ is contained in the set $K(f)$ of generalized critical values of $f$. In this note we study the Rabier function. We give a few equivalent expressions for this function, in particular we compare this function with the Kuo function and with the (generalized) Gaffney function. As a consequence we give a direct short proof of the fact that $f$ is a locally trivial fibration outside the set $K(f)$ (i.e., that $B(f) \subset K(f)$ ). This generalizes the previous results of the author for $X=k^{r}$ (see [2]).
\end{abstract}

1. Introduction. Let $X$ be a smooth affine variety over $k=\mathbb{R}$ or $k=\mathbb{C}$ of dimension $n-r$ and let $f: X \rightarrow k^{m}$ be a polynomial dominant mapping. It is well-known that the mapping $f$ is a locally trivial fibration outside a bifurcation set $B(f)$, which has a measure 0 .

Let us recall that in general the set $B(f)$ is bigger than $K_{0}(f)$ - the set of critical values of $f$. It contains also the set $B_{\infty}(f)$ of bifurcations points at infinity. Briefly speaking, the set $B_{\infty}(f)$ consists of points at which $f$ is not a locally trivial fibration at infinity (i.e., outside a compact set). To control the set $B_{\infty}(f)$ one can use the set of asymptotic critical values at infinity of $f$ (see [6]):

$$
\begin{array}{r}
K_{\infty}(f)=\left\{y \in k^{m}: \text { there is a sequence } x_{l} \rightarrow \infty \text { such that } f\left(x_{l}\right) \rightarrow y\right. \\
\text { and } \left.\left\|x_{l}\right\| \nu\left(\operatorname{res}_{T_{x_{l}} X} d f\left(x_{l}\right)\right) \rightarrow 0\right\},
\end{array}
$$

where we consider the induced Euclidean metric on $X$ and $\nu$ is the function defined by Rabier (see Definition 2.1 below). If $y \notin K_{\infty}(f)$ we say also that $y$ is Malgrange regular.

2000 Mathematics Subject Classification: Primary 51N10; Secondary 15A04.

Research supported by KBN grant 2PO3A 01722.

The paper is in final form and no version of it will be published elsewhere. 
If $m=1$ and $X=k^{n}$, then there is a wide literature devoted to different regularity conditions and their comparison (e.g., [8], [9], [10]). It has been proved for instance that the Malgrange regularity is equivalent to another regularity called t-regularity, by Siersma and Tibăr (see [7]). The case $m>1$ and $X=k^{n}$ was studied in [1], [2] and [4]. In this paper (and in [3]) we study the case when $X$ is a smooth affine variety (or even a Stein submanifold of $\mathbb{C}^{m}$ ) and $m \leq \operatorname{dim} X$.

Let $K(f)=K_{0}(f) \cup K_{\infty}(f)$ be the set of generalized critical values of $f$. It can be proved that the set $K(f)$ is a proper algebraic subset of $\mathbb{C}^{m}$ - or proper semi-algebraic in the real case (see [3]). Moreover, we have (e.g., by a general Fibration Theorem of Rabier [6], see also [1]) $B(f) \subset K(f)$. These two facts together allow us to construct effectively a Zariski open dense subset $U \subset k^{m}$ over which the mapping $f$ is a locally trivial fibration.

In this note we study the Rabier function. As a consequence we give a direct proof of the fact that $B(f) \subset K(f)$ in the case when $X \subset k^{n}$ is a smooth submanifold and $f: X \rightarrow k^{m}$ is a smooth mapping (moreover, some of these results are used in [3] to study the properties of the set $K(f))$.

The fact that $B(f) \subset K(f)$ follows from a very general Theorem of Rabier (see [6]), but it is so important (e.g., in the study of polynomial mappings) that (as I believe) it is worth to have a simple direct proof of it in a special case of submanifolds of a Euclidean space.

Acknowledgments. This paper was written during the author's stay at the MaxPlanck-Institut für Mathematik in Bonn. The author thanks MPI for the invitation and the kind hospitality.

2. On the Rabier function $\nu$. Here we give several equivalent expressions for $\nu$.

Let $X \cong k^{n}, Y \cong k^{m}$ be finite-dimensional vector spaces (over $k$ ). Let us denote by $\mathcal{L}(X, Y)$ the set of linear mappings from $X$ to $Y$ and by $\Sigma(X, Y) \subset \mathcal{L}(X, Y)$ the set of non-surjective mappings. Let us recall the following $([6])$ :

Definition 2.1. Let $A \in \mathcal{L}(X, Y)$. Set

$$
\nu(A)=\inf _{\|\phi\|=1}\left\|A^{*}(\phi)\right\|,
$$

where $A^{*}: \mathcal{L}\left(Y^{*}, X^{*}\right)$ is the adjoint operator and $\phi \in Y^{*}$.

In [4] the following characterization of $\nu$ is given: $\nu(A)=\operatorname{dist}(A, \Sigma)=\inf _{B \in \Sigma}\|A-B\|$. Moreover, we have the following useful characterization ([6] and [4]):

Proposition 2.1. Let $A \in \mathcal{L}(X, Y)$. Then

a) $\nu(A)=\sup \{r>0: B(0, r) \subset A(B(0,1))\}$, where $B(0, r)=\{x \in X:\|x\| \leq r\}$.

b) if $A \in G L(X, Y)$ then $\nu(A)=\left\|A^{-1}\right\|^{-1}$.

Proposition 2.2. Let $A=\left(A_{1}, \ldots, A_{m}\right) \in \mathcal{L}(X, Y)$ and let $\overline{A_{i}}=\operatorname{grad} A_{i}$. Let

$$
\kappa(A)=\min _{1 \leq i \leq m} \operatorname{dist}\left(\overline{A_{i}},\left\langle\left(\overline{A_{j}}\right)_{j \neq i}\right\rangle\right)
$$

be the Kuo number of $A$. Then $\nu(A) \leq \kappa(A) \leq \sqrt{m} \nu(A)$. 
We say that $\nu(A)$ and $\kappa(A)$ are equivalent and write $\nu(A) \sim \kappa(A)$. The symbol $X \sim Y$ means that there are positive constants $C_{1}, C_{2}$ such that $C_{1} X \leq Y \leq C_{2} X$.

Definition 2.2. Let $A \in \mathcal{L}(X, Y)$ and let $H \subset X$ be a linear subspace. We set

$$
\nu(A, H)=\nu\left(\operatorname{res}_{H} A\right), \quad \kappa(A, H)=\kappa\left(\operatorname{res}_{H} A\right),
$$

where $\operatorname{res}_{H} A$ denotes the restriction of $A$ to $H$.

From Proposition 2.2 we get immediately:

Corollary 2.1. Let $A \in \mathcal{L}(X, Y)$ and let $H \subset X$ be a linear subspace. Then

$$
\nu(A, H) \sim \kappa(A, H) .
$$

Proposition 2.3. Let $A=\left(A_{1}, \ldots, A_{m}\right) \in \mathcal{L}(X, Y)$ and let $H \subset X$ be a linear subspace. Assume that $H$ is given by a system of linear equations $B_{j}=0, j=1, \ldots, r$. Then

$$
\kappa(A, H)=\min _{1 \leq i \leq m} \operatorname{dist}\left(\overline{A_{i}},\left\langle\left(\overline{A_{j}}\right)_{j \neq i} ;\left(\overline{B_{j}}\right)_{j=1, \ldots, r}\right\rangle\right),
$$

where $\overline{A_{i}}=\operatorname{grad} A_{i}$ and $\overline{B_{j}}=\operatorname{grad} B_{j}$.

Proof. Indeed, every vector $\overline{A_{i}}$ can be written as $a_{i}+b_{i}$, where $a_{i}$ is orthogonal to the subspace $B=\left\langle\left(\overline{B_{j}}\right)_{j=1, \ldots, r}\right\rangle$ (which means that $a_{i} \in H$ ) and $b_{i} \in B$. Hence

$$
\operatorname{dist}\left(\overline{A_{i}},\left\langle\left(\overline{A_{j}}\right)_{j \neq i} ;\left(\overline{B_{j}}\right)_{j=1, \ldots, r}\right\rangle\right)=\operatorname{dist}\left(a_{i},\left\langle\left(a_{j}\right)_{j \neq i}\right\rangle\right)
$$

and since $\operatorname{grad}\left(\operatorname{res}_{H} A_{i}\right)=a_{i}$, the proof is finished.

We need also:

Definition 2.3. Let $A \in \mathcal{L}(X, Y)$ (where $n \geq m+r$ ) and let $H \subset X$ be a linear subspace given by a system of independent linear equations $B_{i}=\sum b_{i j} x_{j}, i=1, \ldots, r$. Let $\mathbf{a}=\left[a_{i j}\right]$ be the matrix of $A$. Let $\mathbf{c}=\left[c_{k l}\right]$ be a $((m+r) \times n)$ matrix given by the rows $A_{1}, \ldots, A_{m} ; B_{1}, \ldots, B_{r}$ (we identify $A_{i}=\sum a_{i j} x_{j}$ with the vector $\left(a_{i 1}, \ldots, a_{i n}\right)$, similarly for $\left.B_{j}\right)$. Let $M_{I}$, where $I=\left(i_{1}, \ldots, i_{m+r}\right)$, denote a $((m+r) \times(m+r))$ minor of $\mathbf{c}$ given by columns indexed by $I$ and let $\left|M_{I}\right|$ denote the determinant of $M_{I}$. Further, let $M_{J}(j)$ denote a $((m+r-1) \times(m+r-1))$ minor given by columns indexed by $J$ and by deleting the $j$-th row, where $1 \leq j \leq m$. Then by the generalized Gaffney function of $A$ with respect to a linear subspace $H$, we mean the number

$$
g(A, H)=\frac{\left(\sum_{I}\left|M_{I}\right|^{2}\right)^{1 / 2}}{\left(\sum_{J, 1 \leq j \leq m}\left|M_{J}(j)\right|^{2}\right)^{1 / 2}} .
$$

(If this number is not defined we put $g(A, H)=0$.)

REmark 2.1. It is easy to see that $g(A, H)$ depends on $A$ and $H$ only. A particular case of this definition (for $H=X$ ) has been considered by Gaffney-see [1].

Proposition 2.4. Let $A \in \mathcal{L}(X, Y)$ (where $n \geq m$ ) and let $H \subset X$ be a linear subspace. Then $g(A, H) \sim \kappa(A, H) \sim \nu(A, H)$. 
Proof. By basic properties of the Gram determinant (see e.g., [5]) we have

$$
\begin{gathered}
\operatorname{dist}\left(\overline{A_{i}},\left\langle\left(\overline{A_{j}}\right)_{j \neq i} ;\left(\overline{B_{j}}\right)_{j \in\{1, \ldots, r\}}\right\rangle\right)=\frac{G\left(\left(\overline{A_{j}}\right)_{j \in\{1, \ldots, m\}},\left(\overline{B_{j}}\right)_{j \in\{1, \ldots, r\}}\right)^{1 / 2}}{G\left(\left(\overline{A_{j}}\right)_{j \neq i},\left(\overline{B_{j}}\right)_{j \in\{1, \ldots, r\}}\right)^{1 / 2}} \\
=\frac{\left(\sum_{I}\left|M_{I}\right|^{2}\right)^{1 / 2}}{\left(\sum_{J}\left|M_{J}(i)\right|^{2}\right)^{1 / 2}} .
\end{gathered}
$$

Thus $g(A, H) \leq \kappa(A, H)$. On the other hand there is a number $i_{0}$ such that the sum $\left(\sum_{J}\left|M_{J}\left(i_{0}\right)\right|^{2}\right)^{1 / 2}$ is maximal. Since

$$
\left(\sum_{J, j}\left|M_{J}(j)\right|^{2}\right)^{1 / 2}=\left(\sum_{r}\left(\sum_{J}\left|M_{J}(r)\right|^{2}\right)\right)^{1 / 2} \leq \sqrt{m}\left(\sum_{J}\left|M_{J}\left(i_{0}\right)\right|^{2}\right)^{1 / 2},
$$

we have

$$
g(A, H) \geq C \frac{\left(\sum_{I}\left|M_{I}\right|^{2}\right)^{1 / 2}}{\left(\sum_{J}\left|M_{J}\left(i_{0}\right)\right|^{2}\right)^{1 / 2}}=C \operatorname{dist}\left(\bar{A}_{i_{0}},\left\langle\left(\bar{A}_{j}\right)_{j \neq i_{0}} ;\left(\overline{B_{j}}\right)_{j \in\{1, \ldots, r\}}\right\rangle\right) \geq C \kappa(A, H),
$$

where $C=1 / \sqrt{m}$.

Definition 2.4. Let us apply the notation from Definition 2.3. Put

$$
q(A, H)=\frac{\max _{I}\left|M_{I}\right|}{\max _{I, J \subset I, j}\left|M_{J}(j)\right|},
$$

(where we consider only numbers with $M_{J}(j) \neq 0$, if all numbers $M_{J}(j)$ are zero, we put $q(A, H)=0)$.

Proposition 2.4 can also be formulated in the following way:

Corollary 2.2. We have $q(A, H) \sim \nu(A, H)$.

Proof. Let $A$ denote the number of all possible matrices of type $M_{I}$ (for all $I$ ) and let $B$ denote the number of all possible matrices of type $M_{J}(j)$ (for all possible $I, J \subset I$ and all $1 \leq j \leq m)$. Since the norms $\|x\|=\left(\sum\left|x_{i}\right|^{2}\right)^{1 / 2}$ and $\|x\|^{\prime}=\sum\left|x_{i}\right|$ are equivalent, we have

On the other hand

$$
g(A, H) \sim \frac{\sum_{I}\left|M_{I}\right|}{\sum_{I, J \subset I, j}\left|M_{J}(j)\right|} .
$$

$$
(1 / B) \frac{\max _{I}\left|M_{I}\right|}{\max _{I, J \subset I, j}\left|M_{J}(j)\right|} \leq \frac{\sum_{I}\left|M_{I}\right|}{\sum_{I, J \subset I, j}\left|M_{J}(j)\right|} \leq A \frac{\max _{I}\left|M_{I}\right|}{\max _{I, J \subset I, j}\left|M_{J}(j)\right|}
$$

and consequently $g(A, H) \sim q(A, H)$. Now we finish the proof by Proposition 2.4.

At the end of this section we introduce another important function (the notation is as in Definition 2.3):

DEFINITION 2.5. We define the function

$$
g^{\prime}(A, H)=\max _{I}\left\{\min _{J \subset I, 1 \leq j \leq m} \frac{\left|M_{I}\right|}{\left|M_{J}(j)\right|}\right\},
$$

(where we consider only numbers with $M_{J}(j) \neq 0$, if all numbers $M_{J}(j)$ are zero, we put $\left.g^{\prime}(A, H)=0\right)$. 
Proposition 2.5. We have $g^{\prime}(A, H) \sim g(A, H)$.

Proof. First we prove that there is a constant $C>0$ such that $g^{\prime}(A, H) \leq C g(A, H)$. Let us fix an index $I=\left(i_{1}, \ldots, i_{m+r}\right)$ such that $\left|M_{I}\right| \neq 0$ and consider the numbers $\left|M_{I}\right| /\left|M_{J}(s)\right|$, where $J \subset I$ and $1 \leq s \leq m$. For simplicity we can assume that $I=$ $(1, \ldots, m+r)$. Let the subspace $H$ be given by a system of independent linear equations $B_{i}=\sum b_{i j} x_{j}, i=1, \ldots, r$, and let $\mathbf{a}=\left[a_{i j}\right]$ be the matrix of $A$.

Consider the system of linear equations:

$$
\begin{aligned}
\sum_{j=1}^{n} a_{1 j} x_{j} & =y_{1}, \\
\ldots & \ldots \\
\sum_{j=1}^{n} a_{m j} x_{j} & =y_{m}, \\
\sum_{j=1}^{n} b_{1 j} x_{j} & =0, \\
\ldots & \cdots \\
\sum_{j=1}^{n} b_{r j} x_{j} & =0 \\
x_{m+r+1} & =0, \\
\ldots & \quad \cdots \\
x_{n} & =0 .
\end{aligned}
$$

We can solve this system using the Cramer rules. Let $M_{k i}:=M_{J}(i)$ for $J=I \backslash\{k\}$. We have

$$
\begin{aligned}
x_{1} & =\sum_{k=1}^{m}(-1)^{1+k} y_{k} M_{1 k} / M_{I}, \\
\ldots & \quad \cdots \\
x_{m+r} & =\sum_{k=1}^{m}(-1)^{m+r+k} y_{k} M_{(m+r) k} / M_{I}, \\
x_{m+r+1}= & 0 \\
\ldots & \cdots \\
x_{n}= & 0 .
\end{aligned}
$$

In particular we have $\|x\| \leq\left(\max \left|M_{J}(i)\right| /\left|M_{I}\right|\right)|| y \|$. Consequently we see that the image of a unit ball in the subspace $H^{\prime}=\left\{x \in H: x_{m+r+1}=0, \ldots, x_{n}=0\right\}$ by the mapping $A$ contains a ball of radius $\min _{J \subset I, 1 \leq j \leq m}\left|M_{I}\right| /\left|M_{J}(j)\right|$. Now by Proposition 2.1a), we see that $\min _{J \subset I, 1 \leq j \leq m}\left|M_{I}\right| /\left|M_{J}(j)\right| \leq \nu\left(A, H^{\prime}\right) \leq \nu(A, H)$. Finally we get

$$
\nu(A, H) \geq \max _{I}\left\{\min _{J \subset I, 1 \leq j \leq m} \frac{\left|M_{I}\right|}{\left|M_{J}(j)\right|}\right\}=g^{\prime}(A, H) .
$$

In particular there is a constant $C$ such that $C g(A, H) \geq g^{\prime}(A, H)$.

On the other hand, there exists $I_{0}$ such that the minor $M_{I_{0}}$ has a maximal norm. 
Since

$$
\begin{gathered}
g(A, H)=\frac{\left(\sum_{I}\left|M_{I}\right|^{2}\right)^{1 / 2}}{\left(\sum_{J, j}\left|M_{J}(j)\right|^{2}\right)^{1 / 2}} \leq\left(\begin{array}{c}
n \\
m+r
\end{array}\right)^{1 / 2} \frac{\left|M_{I_{0}}\right|}{\left(\sum_{J, j}\left|M_{J}(j)\right|^{2}\right)^{1 / 2}} \\
\leq\left(\begin{array}{c}
n \\
m+r
\end{array}\right)^{1 / 2} \min _{J \subset I_{0}, 1 \leq j \leq m} \frac{\left|M_{I_{0}}\right|}{\left|M_{J}(j)\right|} \leq\left(\begin{array}{c}
n \\
m+r
\end{array}\right)^{1 / 2} g^{\prime}(A, H),
\end{gathered}
$$

we deduce that there is a constant $C^{\prime}>0$ such that $g(A, H) \leq C^{\prime} g^{\prime}(A, H)$.

Corollary 2.3. We have $g^{\prime}(A, H) \sim \nu(A, H)$.

3. Main result. In this section we give a short direct proof of the fact $B(f) \subset K(f)$ for a smooth mapping $f: X \rightarrow k^{m}$, where $X$ is a smooth submanifold of $k^{m}$. Let us recall the following basic definition:

Definition 3.1. Let $k=\mathbb{C}$ or $k=\mathbb{R}$ and let $X$ be a smooth submanifold of $k^{n}$. Let $f: X \rightarrow k^{m}$ be a $k$-smooth mapping. Then we define the set of generalized critical values $K(f)=K_{0}(f) \cup K_{\infty}(f)$, where $K_{0}(f)$ is the set of critical values of $f$ and

$K_{\infty}(f)=\left\{y \in k^{m}:\right.$ there is a sequence $x_{l} \rightarrow \infty$ such that $f\left(x_{l}\right) \rightarrow y$

$$
\text { and } \left.\left\|x_{l}\right\| \nu\left(d f\left(x_{l}\right), T_{x_{l}} X\right) \rightarrow 0\right\}
$$

is the set of critical values at infinity.

REMARK 3.1. Note that by virtue of results of Section 2, in place of the function $\nu$ above we can put also $\kappa, g, q$ or $g^{\prime}$.

We have the following simple observation (see [2], [6]):

Proposition 3.1. Let $k=\mathbb{C}$ or $k=\mathbb{R}$ and let $X$ be a smooth affine variety over $k$. Let $f: X \rightarrow k^{m}$ be a $k$-smooth mapping. Then the set $K(f)=K_{0}(f) \cup K_{\infty}(f)$ is closed.

We need also the following lemma (see [2]):

LEMMA 3.1. Let $U \subset k^{n}$ be an open set and $V: U \rightarrow k^{n}$ be a smooth mapping. Let $y \in U$ and let

$$
x^{\prime}(t)=V(x), \text { with } x(0)=y
$$

be a differential equation. Let $x(y, t), t \in\left[0, t_{0}\right)$, be a solution of this equation. Assume that for $\|x(y, t)\|$ large enough, we have $\|V(x(y, t))\|<M\|x(y, t)\|$. Then this trajectory is bounded. In particular this trajectory either is defined for every $t>0$ or intersects the boundary $\partial U$ of $U$.

Now we give a short direct proof of the fact that $B(f) \subset K(f)$, which is a particular version of a very general result of Rabier [6] (see also [1]).

TheOREM 3.1. Let $k=\mathbb{C}$ or $k=\mathbb{R}$ and let $X \subset k^{n}$ be a smooth submanifold (i.e., $X$ is smooth for $k=\mathbb{R}$ or Stein for $k=\mathbb{C}$ ). Let $f: X \rightarrow k^{m}$ be a $k$-smooth mapping (i.e., $f$ is smooth for $k=\mathbb{R}$ or holomorphic for $k=\mathbb{C}$ ). Then

$$
B(f) \subset K(f)=K_{0}(f) \cup K_{\infty}(f),
$$

i.e., the mapping $f$ is a locally trivial fibration outside the set $K(f)$. 
Proof. It is well-known that we can assume that $f$ can be extended to a $k$-smooth mapping $\bar{f}$ on the whole $k^{n}$ (in real case it is an easy exercise, in complex it follows from the theory of Stein manifolds).

First assume that $X$ is a global complete intersection, i.e. $X=\left\{b_{1}=0, \ldots, b_{r}=0\right\}$ and $\operatorname{rank}\left\{d_{x} b_{1}, \ldots, d_{x} b_{r}\right\}=r$ for every $x \in X$.

Let $a \notin K(f)$. Without loss of generality we can assume that $a=0$. We have $a \notin K_{0}(f)$ and $a \notin K_{\infty}(f)$. This implies that there are $R>0, \epsilon>0, \eta>0$, such that for every $x \in X$ with $\|x\| \geq R$ and $\|f(x)\|<\eta$, we have

$$
\max _{I}\left\{\min _{J \subset I, 1 \leq j \leq m}\|x\| \frac{\left|M_{I}\right|}{\left|M_{J}(j)\right|}\right\}>\epsilon .
$$

Moreover, there is $\omega>0$ such that for every $x \in X$ with $\|x\| \leq R$ and $\|f(x)\|<\eta$, we have $\max _{I}\left|M_{I}(x)\right| \geq \omega$.

Let $U=\left\{y \in k^{m}:\|y\|<\eta\right\}$ and let $\Gamma=f^{-1}(0)$. We show that $f^{-1}(U) \cong \Gamma \times U$ and $f$ is a projection $\Gamma \times U \ni(\gamma, u) \mapsto u \in U$. Indeed, let us define a set

$$
\begin{gathered}
U_{I}=\left\{x \in \bar{f}^{-1}(U): \text { if }\|x\| \geq R \text { then } \min _{J \subset I, 1 \leq j \leq m}\|x\| \frac{\left|M_{I}\right|}{\left|M_{J}(j)\right|} \geq \epsilon,\right. \\
\text { if } \left.\|x\| \leq R \text { then }\left|M_{I}(x)\right| \geq \omega\right\} .
\end{gathered}
$$

Further, let

$$
\begin{gathered}
V_{I}=\left\{x \in \bar{f}^{-1}(U): \text { if }\|x\| \geq R \text { then } \min _{J \subset I, 1 \leq j \leq m}\|x\| \frac{\left|M_{I}\right|}{\left|M_{J}(j)\right|} \leq \epsilon / 2,\right. \\
\text { if } \left.\|x\| \leq R \text { then }\left|M_{I}(x)\right| \leq \omega / 2\right\} .
\end{gathered}
$$

The sets $V_{I}$ and $U_{I}$ are disjoint. Consequently there is a $C^{\infty}$ function $\delta_{I}: k^{n} \rightarrow[0,1]$, which is equal to 1 on $U_{I}$ and to 0 on $V_{I}$. It is easy to see that the sets $H_{I}=\left\{x: \delta_{I}(x)>0\right\}$ cover the set $f^{-1}(U)$. Now take $\delta:=\sum_{I} \delta_{I}$ and let $\Delta_{I}=\delta_{I} / \delta$.

Take $y=\left(y_{1}, \ldots, y_{n}\right) \in U$. Take the index $I=(1, \ldots, m+r)$ and consider a (formal) system of differential equations:

$$
\begin{aligned}
\sum_{j=1}^{n} \frac{\partial \bar{f}_{1}}{\partial x_{j}}(x(t)) x_{j}(t)^{\prime} & =y_{1}, \\
\ldots \ldots & \quad \ldots \\
\sum_{j=1}^{n} \frac{\partial \bar{f}_{m}}{\partial x_{j}}(x(t)) x_{j}(t)^{\prime} & =y_{m} \\
\sum_{j=1}^{n} \frac{\partial b_{1}}{\partial x_{j}}(x(t)) x_{j}(t)^{\prime} & =0 \\
\ldots & \quad \ldots \\
\sum_{j=1}^{n} \frac{\partial b_{r}}{\partial x_{j}}(x(t)) x_{j}(t)^{\prime} & =0 \\
x_{m+r+1}(t)^{\prime} & =0 \\
\ldots & \quad \cdots \\
x_{n}(t)^{\prime} & =0
\end{aligned}
$$


We can solve this system using the Cramer rules (at least in $U_{I}$ ). Let $M_{k i}:=M_{J}(i)$ for $J=I \backslash\{k\}$. We have

$$
\begin{aligned}
x_{1}(t)^{\prime}= & \sum_{k=1}^{m}(-1)^{1+k} y_{k} M_{1 k} / M_{I}, \\
\ldots & \cdots \cdots \\
x_{m+r}(t)^{\prime}= & \sum_{k=1}^{m}(-1)^{m+r+k} y_{k} M_{(m+r) k} / M_{I}, \\
x_{m+r+1}(t)^{\prime}= & 0, \\
\ldots & \cdots \\
x_{n}(t)^{\prime}= & 0 .
\end{aligned}
$$

We can write this system shortly as

$$
x(t)^{\prime}=V_{I}(y, x(t)) .
$$

By the Cramer rules, we have $d f\left(V_{I}(y, x)\right)=y$ and $d b\left(V_{I}(y, x)\right)=0$. In an analogous way we can define $V_{I}$ for an arbitrary index $I=\left(i_{1}, \ldots, i_{m}\right)$.

Now consider a vector field $V(y, x)=\sum_{I} \Delta_{I} V_{I}(y, x)$ in a domain $\bar{f}^{-1}(U)$. By the construction, we have $\|V(x)\| \leq 2 m \eta / \epsilon|| x \|$ for $\|x\| \geq R$ and $x \in X$. Let us consider the differential equation

$$
x(t)^{\prime}=V(y, x(t)), \quad x(0)=\gamma,
$$

where $\gamma \in \Gamma$. Let us note that

$$
\begin{aligned}
d f(V(y, x))=d f\left(\sum_{I} \Delta_{I} V_{I}(y, x)\right)=\sum_{I} d f & \left(\Delta_{I} V_{I}(y, x)\right) \\
& =\sum_{I} \Delta_{I} d f\left(V_{I}(y, x)\right)=\left(\sum_{I} \Delta_{I}\right) y=y .
\end{aligned}
$$

Similarly $d b(V(x, y))=0$. Consequently, if $x(t, y, \gamma)$ is a solution of system (2), then the trajectory is contained in $X$ and $y t=\bar{f}(x(t), y, \gamma)=f(x(t), y, \gamma)$. Since $y \in U$, we see that the trajectory $x(t, y, \gamma), t \in\left[0, t_{0}\right)$ does not cross the border $\partial f^{-1}(U)$ for every $0 \leq t_{0} \leq 1+\delta$, for some $\delta>0$. Consequently by Lemma 3.1 the trajectory $x(t, y, \gamma)$ is defined on the whole $[0,1]$ and is contained in $X$. Since $f(x(t, y, \gamma))=y t$, the phase flow $x(t, y, \gamma), t \in[0,1]$, transforms $f^{-1}(0)=\Gamma$ into $f^{-1}(y)$ (in fact, by the symmetry, it transforms $\Gamma$ onto $\left.f^{-1}(y)\right)$. Let

$$
\Phi: \Gamma \times U \ni(\gamma, y) \mapsto x(1, y, \gamma) \in f^{-1}(U)
$$

It is easy to see that $\Phi$ is a diffeomorphism. Thus $0 \notin B(f)$.

In the general case we can choose a locally finite cover $\left\{U_{i}\right\}$ of $k^{n}$ such that in each $U_{i}$ the manifold $X \cap X_{i}$ is a complete intersection. Now we can construct vector fields $V_{i}$ on $U_{i}$ (construction is as above) and then glue them to one field $V$ by a partition of unity subordinate to the cover $\left\{U_{i}\right\}$. The rest of the proof is the same as above.

At the end of this note we give two simple examples.

ExAmple 3.1. Let us consider a Stein curve $\Gamma=\left\{(x, y) \in \mathbb{C}^{2}: \exp (x y)=2\right\}$. Let us consider the projection $f: \Gamma \ni(x, y) \mapsto y \in \mathbb{C}$. Using the generalized Gaffney function 
we see that

$$
K_{0}(f)=f(\{(x, y) \in \Gamma: y \exp (x y)=0\})=\emptyset
$$

and

$$
K_{\infty}(f)=\left\{\lim f\left(x_{n}, y_{n}\right)=y_{n} ; \text { where }\left(\left|x_{n}\right|+\left|y_{n}\right|\right) \rightarrow \infty \text { and }\left|y_{n}\right| \rightarrow 0\right\}=\{0\} .
$$

Hence finally $K(f)=\{0\}$ and indeed we can check directly that in this case $B(f)=$ $K(f)=\{0\}$ (in fact $f$ is a topological covering outside 0 ). Note that the mapping $f$ has no usual critical values.

EXAMPLE 3.2. Let us consider a smooth mapping

$$
f: \mathbb{C}^{3} \ni(x, y, z) \mapsto(x \exp (z), y \exp (z)) \in \mathbb{C}^{2} .
$$

Using the function $g^{\prime}$ we can easily compute that $K(f)=\{0\}$. But the function $f$ is a global fibration of $\mathbb{C}^{3}$-in fact it gives a fibration

$$
\mathbb{C}^{2} \times \mathbb{C} \ni((x, y), z) \mapsto(x \exp (-z), y \exp (-z), z) \in \mathbb{C}^{3} .
$$

Thus in general $B(f) \neq K(f)$.

\section{References}

[1] T. Gaffney, Fibers of polynomial mappings at infinity and a generalized Malgrange condition, Compositio Math. 119 (1999), 157-167.

[2] Z. Jelonek, On the generalized critical values of a polynomial mapping, Manuscripta Math. 110 (2003), 145-157.

[3] Z. Jelonek, K. Kurdyka, Quantitative Generalized Bertini-Sard Theorem for smooth affine varieties, to appear.

[4] K. Kurdyka, P. Orro, S. Simon, Semialgebraic Sard Theorem for generalized critical values, J. Differential Geom. 56 (2000), 67-92.

[5] A. Mostowski, M. Stark, Elementy Algebry Wyższej, PWN, Warszawa, 1968 (in Polish).

[6] P. J. Rabier, Ehresmann fibrations and Palais-Smale conditions for morphisms of Finsler manifolds, Ann. of Math. (2) 146 (1997), 647-691.

[7] D. Siersma, M. Tibăr, Singularities at infinity and their vanishing cycles, Duke Math. J. 80 (1995), 771-783.

[8] M. Tibăr, On the monodromy fibration of polynomial functions with singularities at infinity, C. R. Acad. Sci. Paris Sér. I Math. 324 (1997), 1031-1035.

[9] M. Tibăr, Regularity at infinity of real and complex polynomial functions, in: Singularity Theory (Liverpool, 1996), London Math. Soc. Lecture Note Ser. 263, Cambridge Univ. Press, Cambridge, 1999, 249-264.

[10] M. Tibăr, Topology at infinity of polynomial mappings and Thom regularity condition, Compositio Math. 111 (1998), 89-109. 